\title{
LXX. On the fitting-up of microscopes for the examination of opaque objects requiring high powers; and on the construction of a Focimeter
}

\author{
Mr. G. Dakin
}

To cite this article: Mr. G. Dakin (1828) LXX. On the fitting-up of microscopes for the examination of opaque objects requiring high powers; and on the construction of a Focimeter , Philosophical Magazine Series 2, 4:24, 429-431, DOI: 10.1080/14786442808674882

To link to this article: http://dx.doi.org/10.1080/14786442808674882

曲 Published online: 10 Jul 2009.

Submit your article to this journal $[\pi$

Џll Article views: 2

Q View related articles $\sqsubset$ 
LXX. On the Fitting-up of Microscopes for the Examination of Opaque Objects requiring high powers; and on the Construction of a Focimeter. By Mr. G. Dakin.

To the Editors of the Philosophical Magazine and Annals. Gentlemen,

A $S$ microscopes are now fitted up by the opticians, a nuA merous and beautiful class of objects is entirely lost to observers; viz. those opaque objects which require a high power. My single microscope, which I had purposely fitted up with the highest powers both opaque and transparent, is almost useless for this purpose, as the highest opaque eye-piece is 1-6th of an inch focus. I have seen the scales of the diamondbeetle as an opaque object through a lens which I think Dr. Goring said was 1-60th of an inch focus. He has certainly carried it quite to the maximum, as there was rather a want of light and distinctness, which I attribute in a great measure to the best part of the speculum being lost by the introduction of the deep convex cup which holds the lens; nevertheless the beautiful lines on the scales were plainly to be seen, even with this high power. I have made the whole of my transparent eyepieces answer as opaque ones, in which the lenses are placed on the outsides of the speculums; consequently the whole cf the central and best part of the speculum is brought into action. Opticians seem to forget that the light which is thrown on the speculum is already condensed, and that such large ones are not necessary, unless it be to compensate for the clumsy manner in which they generally fit up the opaque slides. Objects of this sort ought to be fixed on small cylinders of jet or ebony, and these glued on slips of glass : by this means little or no light is lost. The speculums I made were about $\frac{1}{2}, \frac{3}{8}, \frac{1}{4}, \frac{1}{6}$, and $\frac{1}{8}$ th of an inch diameter: they should be fitted into a brass ring (fig. 1.), to which should be soldered a piece of wire, fitting a hole in a larger piece of wire (fig. 2.), which should slide easily through the stage (fig. 3.); or they may be laid on the slips of glass over the objects (fig. 4.), provided the cylinders are adjusted to bring the objects into the focus of the speculums. When the highest powers are used, take a very small piece of the object and lay it on a small cylinder, which must have a fine hole in the side, for the purpose of fixing it on the point of a fine needle; place the object in the centre of the stage, and bring the speculum down so as to illuminate the object as much as possible. The eye-piece must then be brought down close to the hole in the speculum, the eye being now placed in its right position. The eye-piece may be raised 
to its true focal distance; this will prevent the lenses being injured by coming in contact with the speculum.

The highest power in my microscope is about 1-46th inch focus; but a lens of 1-30th inch focus may be used by a novice with ease and very great advantage, and the object will be as well illuminated as by one of the large speculums. As they are very easily made, I shall describe the method of making a 1-4th inch one. Melt a little fine silver by the blowpipe into a globule about 1-10th of an inch diameter; hammer it out till it is full 1-4th inch wide, and as thick as stout fools.. cap paper : take a piece of brass wire, barely 1-4th inch thick, and file one end of it hemispherically; then lay the silver on a piece of lead about 1-4,th inch thick. Hollow the silver to the shape of the wire, and then drive it quite through the lead, turning the wire at the same time: make a small hole in the centre, and fix it on the end of a stick of sealing-wax; then file a piece of slate-pencil, barely 1-4th inch diameter, to fit the silver cup, and grind them together with fine emery, till it is of a uniform figure quite over the surface; then wash the emery off, and grind the scratches out with the pencil, washing the mud away with clear water. To polish it, cover the pencil with thin silk, rub a little tallow and jeweller's rouge on the silk, and work them together till the speculum is beautifully polished.
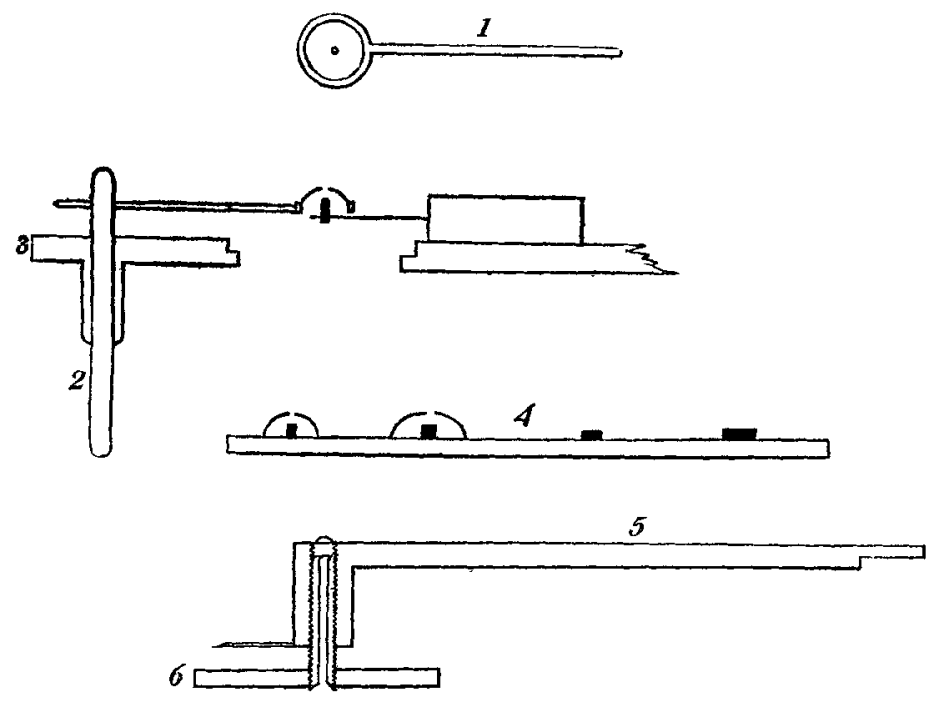

I strongly suspect that the focal distances of lenses are generally 
nerally underrated by opticians. To prove mine, I made what may be called a Focimeter. It consists of a flat piece of brass, which fixes on the arm of the microscope (fig. 5.); at the end is a female screw that has exactly fifty threads to the inch, to which is fitted a male screw about half an inch long, with a hole drilled quite through it, and a large ivory head (fig. 6.), with twenty divisions fixed on its lower end; consequently one division on the head is equal to the 1-1000dth of an inch. After the end of the screw and the brass plate are ground together on a flat hone, there must be glued over the hole in the male screw, a small piece of the outer membrane of the eye of the Libellula grandis, and a piece of very thin foil with a small hole in its centre laid over the female screw. When used, fix it to the arm of the microscope, and throw the light up the hole by the mirror; lay the highest power on the foil, turn the screw up till it just touches the foil, and set down the number. of turns and divisions that it takes to bring the object down to the focus of the lens.

Proceed in the same manner with the rest of the powers; they may then be easily reduced to the nearest fraction having one for a numerator: by this means I found that my highest power, which I considered 1-60th of an inch focus, was only 1-46th inch focus, and the rest in proportion. I have made a double convex lens, which is the smallest $I$ have ever seen, and is only 1-100dth inch focus, as it took but half a turn of the screw to bring the object down to the focus. The thickness of the foil may be neglected even in the highest powers; as it does not amount to the 1-1000dth of an inch.

Dr. Goring uses the scales of the Lepidoptera as test-objects: but the most beautiful and delicate test-objects that I have ever seen are the scales of the Lepisma saccharina; they are so very thin, and the lines upon them are so very fine, that they will bear almost any power. The beautiful green convex scales of the small English diamond-beetle, which is very common in the summer months, is a very good opaque test-object.

The focal distance of large lenses for telescopes, \&c. is reckoned from the centre of the body of the lens (under half an inch focus); but in my opinion the focus of microscopic lenses ought to be computed from the surface next the radiant object, otherwise another operation is necessary; viz. taking the thickness of the lens and adding half of it to the focal distance: but as small lenses are so very apt to be lost or cracked, it is much safer and better to reckon from the surface.

Ninesham, Oct. 23, 1828. I beg to remain yours, \&c. G. DAKIN. 